\title{
Electronic structure and superconductivity of Europium
}

\author{
Lane W. Nixon and D. A. Papaconstantopoulos \\ Department of Computational and Data Sciences, George Mason University, Fairfax, VA 22030
}

(Dated: September 17, 2018)

\begin{abstract}
We have calculated the electronic structure of Eu for the bcc, hcp, and fcc crystal structures for volumes near equilibrium up to a calculated $90 \mathrm{GPa}$ pressure using the augmented-plane wave method in the local-density approximation. The frozen-core approximation was used with a semi-empirical shift of the f-states energies in the radial Schrödinger equation to move the occupied $4 \mathrm{f}$ valence states below the $\Gamma_{1}$ energy and into the core. This shift of the highly localized f-states yields the correct europium phase ordering with lattice parameters and bulk moduli in good agreement with experimental data. The calculated superconductivity properties under pressure for the $b c c$ and $h c p$ structures are also found to agree with and follow a $T_{c}$ trend similar to recent measurement by Debessai et al $\stackrel{1}{\underline{1}}$
\end{abstract}

\section{INTRODUCTION}

A recent paper by Debessai et al. 1 reports on membranedriven diamond-anvil experiments on europium showing the onset and linear increase of superconductivity under hydrostatic pressure. A transition temperature near $1.8 \mathrm{~K}$ was observed near $80 \mathrm{GPa}$, increasing to 2.75 at $142 \mathrm{GPa}$. Previous $\mathrm{X}$-ray diffraction experiments by Takemura and Syassen ${ }^{2}$ note the bcc, hcp, hcp-like crystal structures, with transitions occurring at 12.5 and $18 \mathrm{GPa}$, respectively.

Several theoretical works dating back to the mid 1960s have focused on the electronic structure of the equilibrium bcc structure. Freeman and Dimmock reported the first band structure calculations using the non-self-consistent augmented plane-waves (APW) method ${ }^{3}$ Andersen and Loucks soon followed with relativistic APW (RAPW) method which also was not self consistent and used the same exchange potential parameter $\alpha=1.0 .^{4}$ Kobayasi et al. performed similar, non-self-consistent calculations with the KKR method with $\alpha$ $=1.0,0.9,0.8$ and $0.67 \cdot \frac{5}{}$ Matsumoto et al. related the shape of the Fermi surfaces to helical spin ordering then reported the first self-consistent KKR results for $\alpha=1.0$ and 0.67 , placing the occupied $4 \mathrm{f}$ bands near those from experiment. 6,7. Matsumoto et al. then reported self-consistent paramagnetic band structure using the $\mathrm{X} \alpha$-KKR method.$^{8}$ Later Min et al. performed self-consistent semi-relativistic and fully-relativistic linearized muffin-tin orbital (LMTO) total energy calculations within the local density approximation (LDA) for ambient and high pressure bcc Europium $\stackrel{9}{-}$ Recently Turek et al. calculated the exchange parameters, corresponding to magnon spectrum, and Neel temperature from a real-space perturbation approach derived from the tight-binding linear muffin-tin (TB-LMTO) method. ${ }^{10}$ And Kunes and Laskowski calculated the magnetic ground state and Fermi surface of bcc Europium using the linearized augmented-plane-waves (LAPW) method with $\mathrm{LDA}+\mathrm{U}$ to determine spin spiral states. ${ }^{11}$

This work reports the results of paramagnetic selfconsistent, scalar-relativistic, frozen-core augmented planewave (APW) calculations of the total energies and electronic structures of $6 s^{2}$ valence bcc, fcc, and hcp europium for the Hedin-Lundqvist ${ }^{12}$ local-density approximation (LDA) method with a semi-empirical shift of the f-state energies.

\section{TOTAL ENERGIES}

In the spirit of previous bcc europium spin-polarized calculations that split localized f-states near $\epsilon_{F}$ to determine structural and magnetic properties in bcc europium (see for example Refs. 9.10), self-consistent scalar-relativistic frozen core APW total energy calculations were conducted for the bcc, fcc, and hcp structures with a volume-based empirical shift of the f-states to determine the ground state and superconductivity properties as functions of pressure. The Brillouin-zone summations over the valence states used $285 \mathrm{bcc}, 45 \mathrm{hcp}$, and 505 fcc k-points in the irreducible portion of the Brillouinzone and a broadening temperature equivalent to $2 \mathrm{mRy}$. To simplify the volume-based shift procedure, the hcp structure was only considered as ideal with c/a ratio $\sqrt{ } \frac{8}{3}$. Experiment however shows a decrease in the c/a ratio from 1.60 at 12.5 $\mathrm{GPa}$ to near 1.56 at $18 \mathrm{GPa}^{2}$

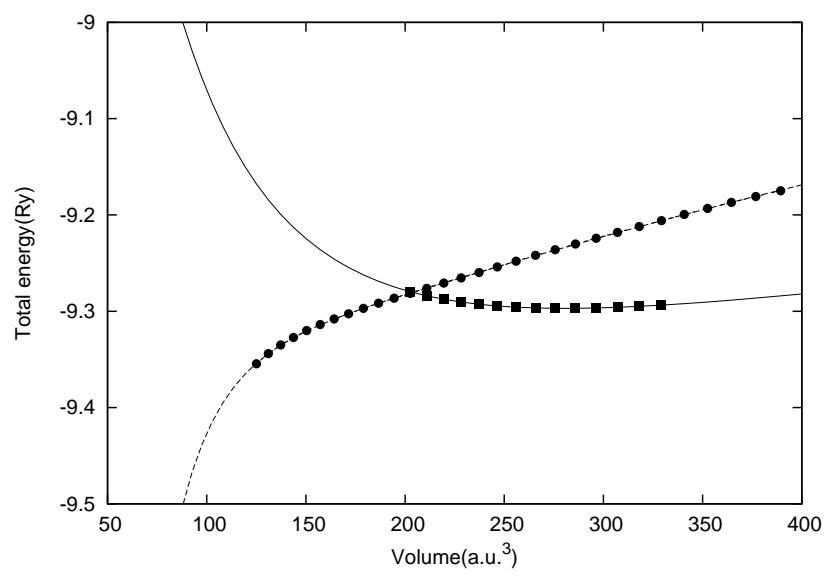

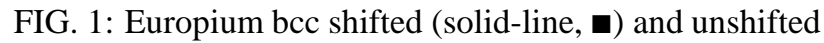
(dashed-line, o) f-states total energy showing no minimum.

In a previous paper on lanthanum Ref. 13 we were able to accurately determine ground state and superconductivity properties because the unoccupied $4 \mathrm{f}$-bands are localized well above the Fermi level. Calculating similar properties for europium is problematic because common approximations to density functional theory (DFT) such as the local density approximation (LDA) as shown in Fig. 1 fail to reliably capture ground state properties due to the highly localized $4 \mathrm{f}$-states below the Fermi level ${ }^{10}$ Figure 1 also shows the bcc LDA to- 
tal energies in which we have applied an empirical shift to the f-states as described in detail below. No energy minimum was found for the unshifted f-states calculation which decrease monotonically in energy with volume.

Since we are most concerned with paramagnetic bulk properties and a reliable ground state, the occupied 4f-states near the Fermi level were placed below the $\Gamma_{1}$ state and treated as part of the core. The remaining two $6 p$ valence electrons per atom were treated as scalar-relativistic bands. The volumebased shift energy of the f-states was determined in the bcc, fcc, and hcp structures at volume ol $_{i}$ with,

$$
E_{f-\text { shift }, \text { vol }=i}=\frac{C_{c r y s t a l}}{\left(\left(\frac{\text { vol }_{i}}{\text { vol }_{\text {ref }}}\right)^{1 / 2} 2 / 3^{1 / 4}\right)},
$$

where $C_{\text {crystal }}$ was the shift coefficient for the bcc, fcc or hcp structure, $v l_{i}$ was the volume for the shifted calculation, and vol $_{\text {ref }}$ was the reference volume. All structures used the same reference volume of $319.20 a u^{3}$ which is the volume for the experimental bcc lattice $\mathrm{a}=8.61 \mathrm{au}$. At this volume the bcc shift coefficient $C_{b c c}$ was $0.6897 \mathrm{Ry}$ while the fcc and hcp shift coefficients, $C_{f c c}$ and $C_{h c p}$ respectively, were both $(2 / 3)^{1 / 2} C_{b c c}$ which was equivalent to $0.5632 \mathrm{Ry}$. The $4 \mathrm{f}-$ energies were shifted in the frozen-core self-consistent iterations by adding the shift energy $\left(E_{f-s h i f t, v o l=i}\right)$ to the f-state energies on the right side of the radial Schrödinger equation:

$$
-\frac{1}{r^{2}} \frac{d}{d r}\left(r^{2} \frac{d u_{l}}{d r}\right)+\left(\frac{l(l+1)}{r^{2}}+V_{n}\right) u_{l}=E u_{l},
$$

where for $l=3, E=E+E_{f-s h i f t, v o l=i}$. The bcc shifts yield the energy minimum as shown in Fig. 1 as the solid-line with black squares.

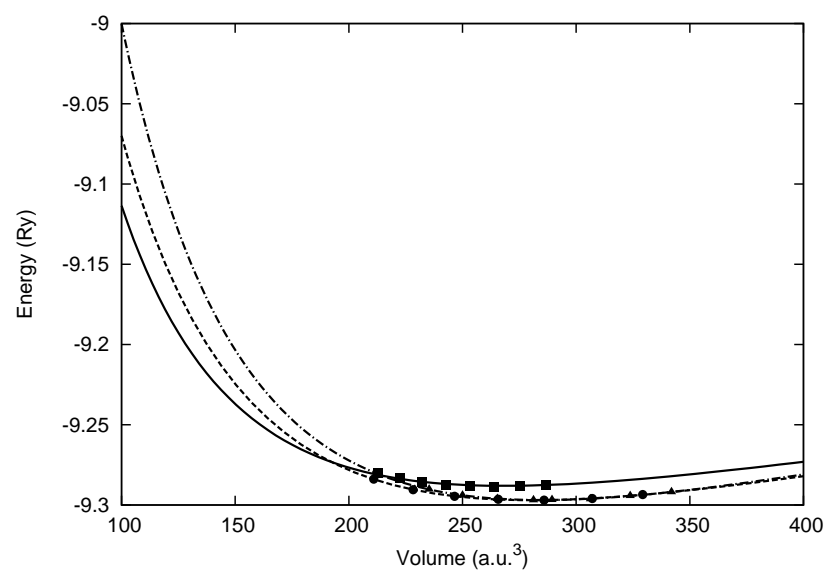

FIG. 2: Europium hcp (solid-line, $\mathbf{})$, bcc (dashed-line, and fcc (dash-dot-line, $\mathbf{\Delta})$ total energies relative to fourth-order Birch fit to bcc structure.

In Fig. 2 we show the total energies for europium hcp, bcc, and fcc using the prescribed shifted f-states as a function of volume. The symbols represent the calculated values and the lines are from the $4^{\text {th }}$ order Birch fit. The bcc ground state is correctly determined with an equilibrium lattice constant of 8.25 bohr which is about $4 \%$ below experiment (typical of LDA calculations) and a calculated bulk modulus of $14.8 \mathrm{GPa}$ that is about $1 \%$ greater than experiment. 14,15 The bcc and fcc total energies are very close with bcc equilibrium about $0.3 \mathrm{mRy}$ below the fcc.

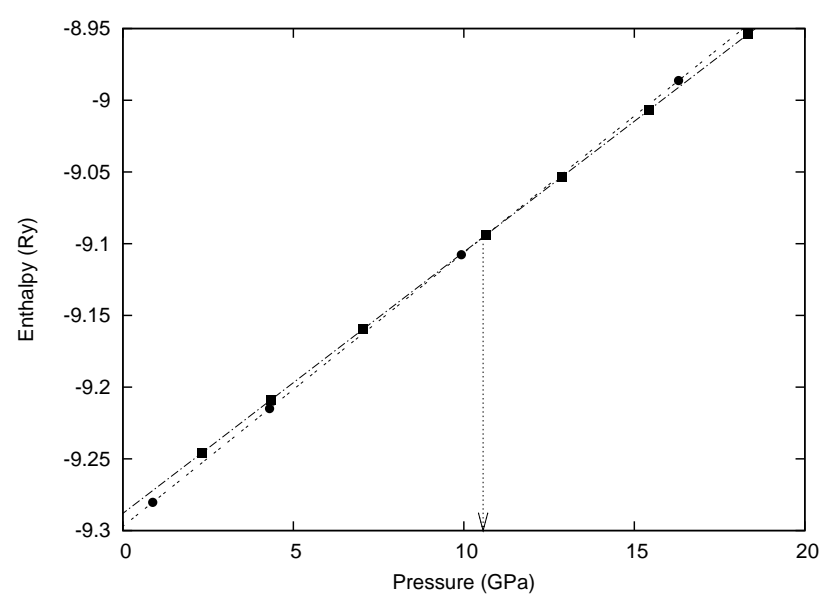

FIG. 3: Europium hcp (solid-line, $\mathbf{a})$ and bcc (dashed-line, ๑) enthalpies.

Since our DFT calculations use temperature $\mathrm{T}=0 \mathrm{~K}$, the enthalpy is equal to the Gibbs free energy $G=E+p V-T S$ such that

$$
H(P)=E(P)+P V,
$$

The calculated enthalpies are shown in Fig. 3 as functions of pressure for the bcc (circles) and hcp (squares) structures. The transition pressure occurs where the $\mathrm{H}(\mathrm{P})$ curves for the bcc and hcp cross near $10.6 \mathrm{GPa}$ which is $15.2 \%$ below the transition observerd at $12.5 \mathrm{GPa}$ in experiment. ${ }^{2}$ Figure 4 compares the calculated volume ratios of the hcp, bcc, and fcc as functions of pressure with the ratios from experiment. 16 Though the calculated volume ratios as a function of pressure follow a trend similar to experiment they are slightly larger.

Our bcc results compare well with previous linearized muffin-tin orbital, calculations of Min et al $\stackrel{\underline{9}}{\text { Their calcula- }}$ tions with the paramagnetic $4 \mathrm{f}$ electrons as valence states severly overestimate the bonding of the localized $4 \mathrm{f}$ band, producing a small equilibrium lattice constant 7.61 au and a large bulk modulus $45 \mathrm{GPa}$. Their ferromagnetic calculations split the $4 \mathrm{f}$ states and generated a lattice constant of 8.24 au and bulk modulus of $25 \mathrm{GPa}$. Placing the $4 \mathrm{f}$-electrons in the core significantly improved the bcc equilibrium lattice $\mathrm{a}=8.73 \mathrm{au}$, which is about $1.4 \%$ above the experimental lattice of 8.61 au for both their paramagnetic and ferromagnetic calculations. However their paramagnetic and ferromagnetic bulk moduli, which are $20 \mathrm{GPa}$ and $19 \mathrm{GPa}$ respectively, are about $33 \%$ larger than the $15 \mathrm{GPa}$ found in experiment. Our empiricalshift model achieves bcc lattice constants similar to those from the ferromagnetic calculations by Min et. al but we also find a bcc equilibrium bulk modulus of $14.8 \mathrm{GPa}$ that is very close to experiment. 


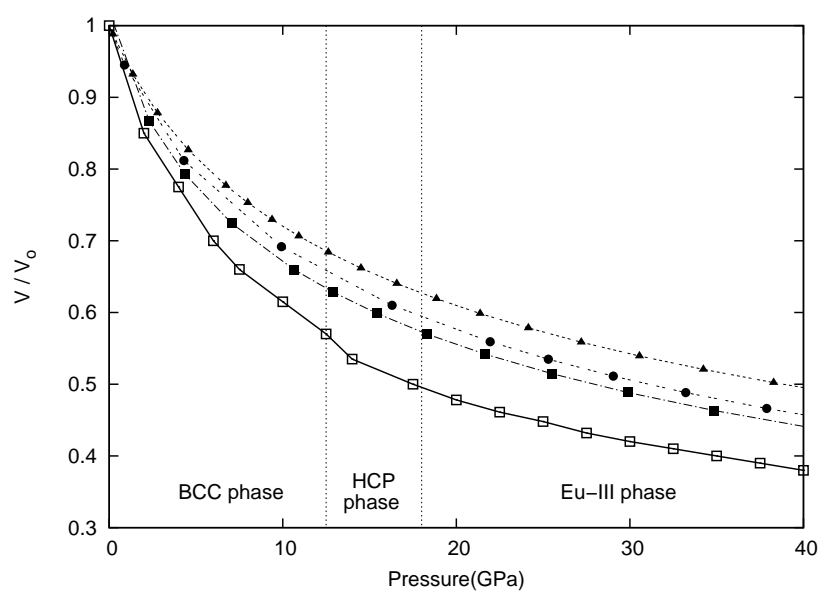

FIG. 4: Europium hcp (solid-line, $\mathbf{0})$, bcc (dashed-line, and fcc (dash-dot-line, $\mathbf{\Delta}$ ) volume ratios as functions of pressure. Experimental data (solid-line, $\square$ ) are from Grosshans et al. (Ref 16).

The results from our first-principles calculations became input to the rigid-muffin-tin approximation (RMTA) theory developed by Gaspari and Gyorffy to yield the high-pressure superconducting behavior of bcc and hcp europium, providing additional insight into the high pressure $T_{c}$ trend of Eu-III. ${ }^{17,18}$

\section{DENSITY OF STATES}

The density of states, $\mathrm{N}(\epsilon)$, were calculated by the tetrahedron method ${ }^{19}$ using the converged self-consistent frozencore bands. The bcc and fcc $\mathrm{N}(\epsilon)$ were determined using 285 and 505 fcc k-points respectively. The hep $\mathrm{N}(\epsilon)$ was calculated from Gillan's method ${ }^{20}$ as described in Ref. 13 but with a larger Fermi temperature of 0.02 Ry to provide additional weight to the broadening function within the k-point mesh. The sum of the product of energy eigenvalues and matrix elements using $45 \mathrm{k}$-points in the irreducible Brillouin zone yielded the weighted hcp density of states. Figures 5 and 6 show the calculated bcc density of states for the shifted and unshifted $4 \mathrm{f}$-energies respectively at equilibium $(\mathrm{a}=8.25$ a.u.).

The DOS that corresponds to the case of the shifted f-states has the Fermi level, $\epsilon_{F}$, located to the right of a peak near $0.3 \mathrm{Ry}$ and has a large d-like contribution. The DOS of the unshifted f-states for a narrow region near $\epsilon_{F}$ are shown in Fig. 6. The occupied $4 \mathrm{f}$-states are located below $\epsilon_{F}$ and the unoccupied $4 \mathrm{f}$-states found above $\epsilon_{F}$. The unshifted f-states total DOS at $\epsilon_{F}$, which is composed almost entirely of f-like states, is about 100 times greater than the shifted $\mathrm{N}\left(\epsilon_{F}\right)$.

The significant influence of the d-like states at $\epsilon_{F}$ is viewed best in Fig. 7, where the ratio $\mathrm{N}\left(\epsilon_{F}\right)_{l} / N\left(\epsilon_{F}\right)$ is displayed as a function of pressure for the hcp (a), bcc (b), and fcc (c) structures. The dominant d-like states remain fairly constant showing only moderate changes with pressure. The hcp d-like

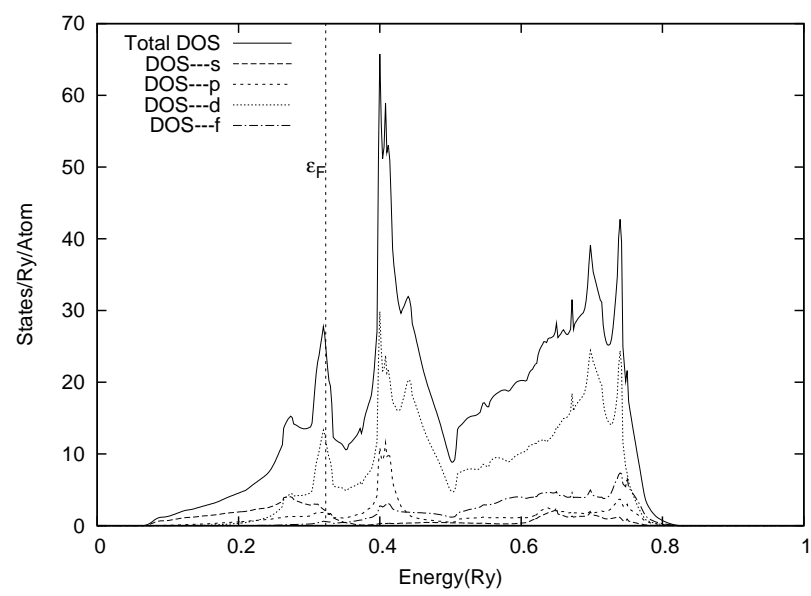

FIG. 5: Europium shifted f-states bcc equilibruim ( $\mathrm{a}=8.25$ a.u.) total and decomposed density of states.

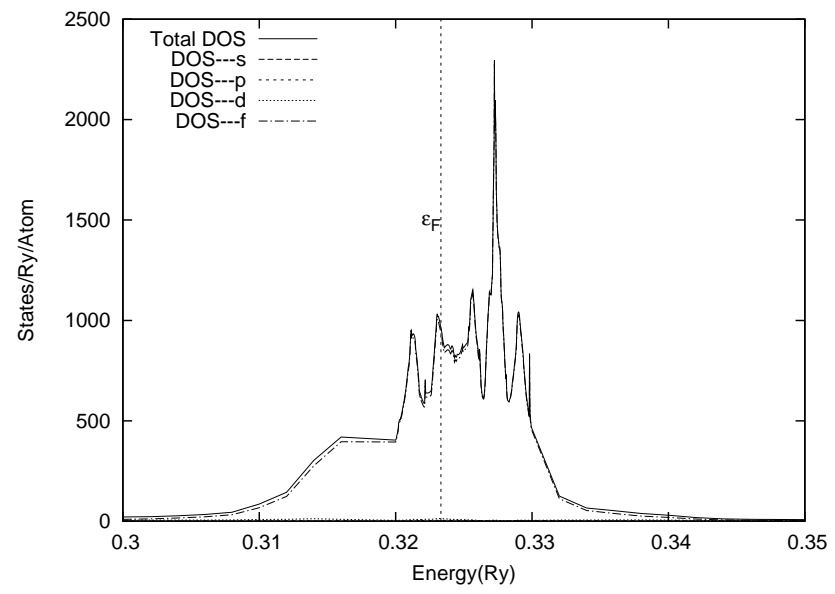

FIG. 6: Europium unshifted f-states bcc equilibrium $(a=8.25$ a.u.) total and decomposed density of states at a narrow range near the Fermi level.

states at $\epsilon_{F}$ increase to almost 0.8 near $24 \mathrm{GPa}$, drop to a low of about 0.61 near $70 \mathrm{GPa}$ then increase at higher pressures. The bcc d-like states at $\epsilon_{F}$ decrease from 0.73 at equilibrium to 0.68 at near $12.5 \mathrm{GPa}$. Between $12.5 \mathrm{GPa}$ and $58 \mathrm{GPa}$ the bcc d-like states increase back to 0.73 then return to about 0.68 near $58 \mathrm{GPa}$. At higher pressures the bcc d-like states increase reaching a maximum of almost 0.78 near $90 \mathrm{GPa}$. And the fcc d-like states at $\epsilon_{F}$ decrease almost monatonically from equilibrium pressure to about 0.60 near $70 \mathrm{GPa}$. The fcc d-like states show only minor increases at higher pressures.

The hcp f-like states at $\epsilon_{F}$ increase monatonically with pressure with relatively no change between 25 and $35 \mathrm{GPa}$ and a slight decrease after about $85 \mathrm{GPa}$. At equilibrium pressure the hcp f-like states are about 0.05 and reach a maximum of almost 0.2 near $85 \mathrm{GPa}$. The bcc f-like states at $\epsilon_{F}$ increases from about 0.05 at equilibrium to almost 0.1 near $58 \mathrm{GPa}$, de- 


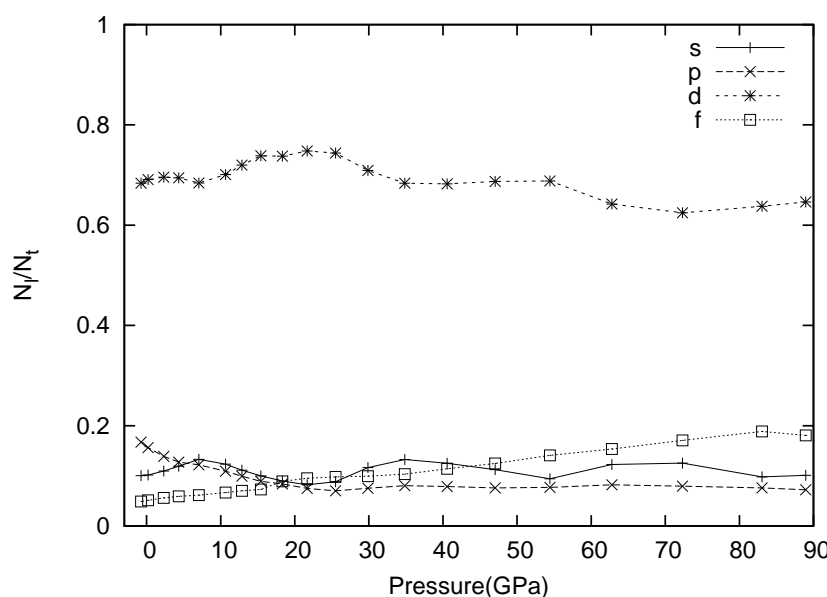

(a) hcp $N_{l}\left(\epsilon_{F}\right) / N_{t}\left(\epsilon_{F}\right)$

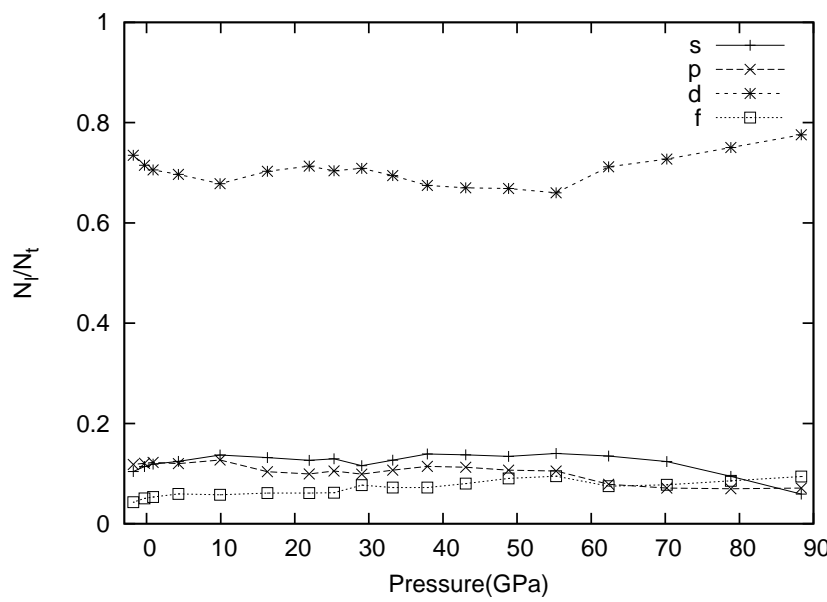

(b) bcc $N_{l}\left(\epsilon_{F}\right) / N_{t}\left(\epsilon_{F}\right)$

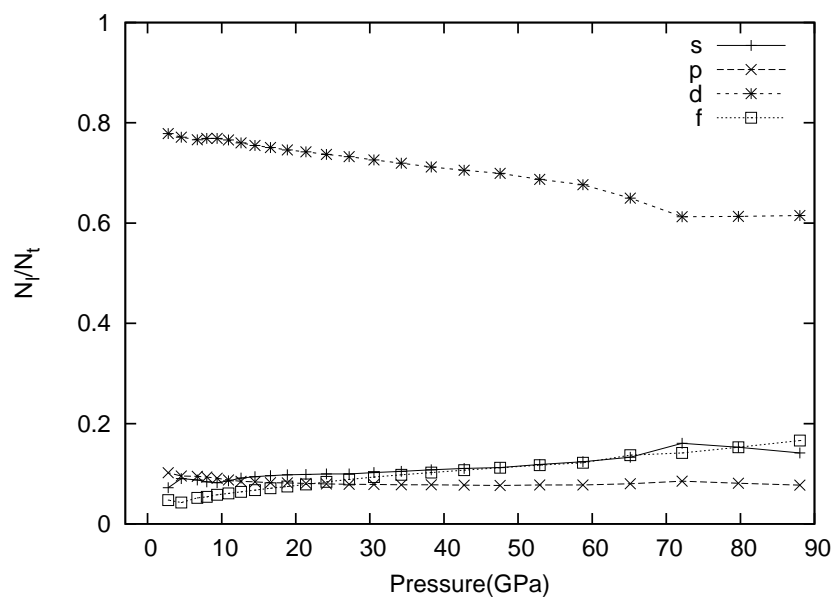

(c) fcc $N_{l}\left(\epsilon_{F}\right) / N_{t}\left(\epsilon_{F}\right)$

FIG. 7: Europium (a) hcp, (b) bcc, and (c) fcc angular momentum decomposed DOS divided by total DOS at $\epsilon_{F}$.

creases to about 0.07 above $60 \mathrm{GPa}$, and increases back to almost 0.1 near $90 \mathrm{GPa}$. The fcc f-like states at $\epsilon_{F}$ increase monatonically with pressure from about 0.035 near $4 \mathrm{GPa}$ to almost 0.17 near $90 \mathrm{GPa}$.

The hcp s-like density of states at $\epsilon_{F}$ oscillate with pressure from a high of almost 0.16 to a low near 0.1 . The bcc s-like states at $\epsilon_{F}$ show only minor changes from equilibrium pressure to about $62 \mathrm{GPa}$. Afterwards they decrease to almost 0.06 near $90 \mathrm{GPa}$. The fcc s-like states increase monatonically from almost 0.06 near $4 \mathrm{GPa}$ to about 0.15 slightly with pressure to equilibrium where is remains relatively constant with pressure.

The hcp p-like $\mathrm{N}\left(\epsilon_{F}\right)$ ratio decreases from about 0.18 at equilibrium to about 0.09 at $20 \mathrm{GPa}$ where it shows no change with pressure. The bcc p-like $\mathrm{N}\left(\epsilon_{F}\right)$ decreases slowly from near 0.12 at equilibrium to about 0.06 near $62 \mathrm{GPa}$, remaining constant with pressure. And the fcc p-like $\mathrm{N}(\epsilon)$ remains constant with pressure from about 0.08 near $4 \mathrm{GPa}$.

Overall the $N_{l}\left(\epsilon_{F}\right) / N_{t}\left(\epsilon_{F}\right)$ ratios vary slowly with pressure.

\section{A. Band Structure}

Figure 8 shows the band structures of bcc equilibrium respectively with the shift of the energy of the 4f-states (Fig.8a) and without the shift (Fig. 8b). The shifted 4 f-states are below the $\Gamma_{1}$ energy and highly localized within a energy block less than 0.13 Ry. The unshifted 4f-states are still highly localized but entangle themselves near the Fermi level, making it impossible to determine the minimum energy for the structure.

Our empirical-shift model explicitly omits hybridization of the occupied $4 \mathrm{f}$ levels by placing them below the $\Gamma_{1}$ level, they contribute little to chemical bonding. ${ }^{21}$ Spin-polarized calculations by Kunes and Laskowski, Min et al., and others that segregate the occupied and unoccupied $4 \mathrm{f}$ states respectively below and above $\epsilon_{F}$ report negligible mixing of the occupied 4f states. ${ }^{9,11}$ Recent photoemission studies by Wang et al. reports the highly localized 4 f core level at about 0.154 Ry below the Fermi level. Replicating the 4 f core location with such proximity to $\epsilon_{F}$ would be difficult even with the more sophisticated LDA $+\mathrm{U}$ or the self-interaction corrected (SIC) LSDA approaches. 


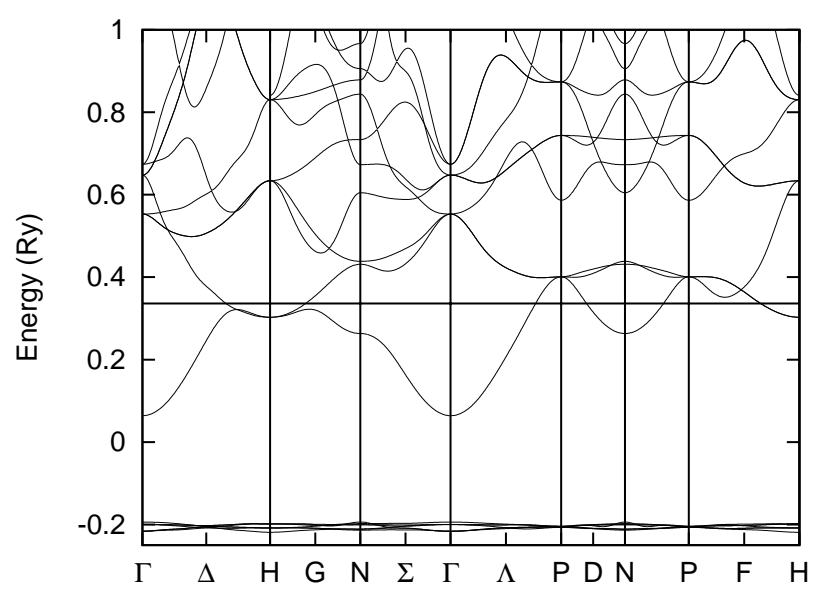

(a) Shifted 4f-states

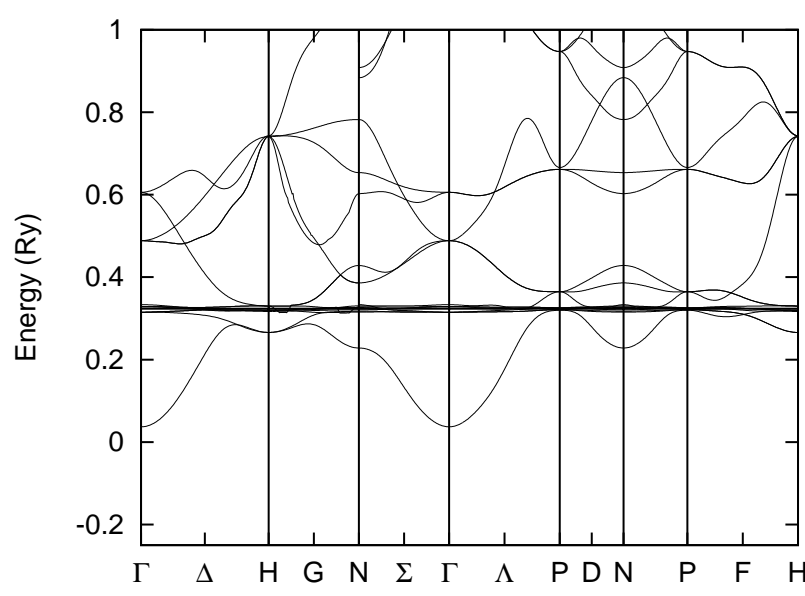

(b) Unshifted $4 \mathrm{f}$-states

FIG. 8: Europium equilibrium bcc shifted and unshifted f-states band structures.

\section{SUPERCONDUCTIVITY}

From our densities of states results at the Fermi level we determine, from first-principles, the electron-phonon coupling constant $\lambda$ and the superconducting transition temperature $T_{c}$. Using McMillan's strong coupling theory, ${ }^{18}$ we take the electron-phonon coupling constant by the following formula:

$$
\lambda=\frac{\eta}{M<\omega^{2}>},
$$

where $\mathrm{M}$ is the atomic mass, $\eta$ is the Hopfield parameter ${ }^{22}$ calculated with the RMTA, ${ }^{17,18} \mathrm{~N}\left(\epsilon_{F}\right)$ is the total density of states at the Fermi level, $\epsilon_{F},\left\langle I^{2}>\right.$ is the square of the electron-ion matrix element at $\epsilon_{F}$, and $<\omega^{2}>$ is the square of the average phonon frequency. The Hopfield parameter is determined by the quantity

$$
\eta=N\left(\epsilon_{F}\right)<I^{2}>,
$$

using the RMTA. The square matrix element $\left\langle I^{2}>\right.$, which is derived from multiple-scattering theory and is also determined by the RMTA proposed by Gaspari and Gyorffy, is defined by the formula

$$
<I^{2}>=\frac{\epsilon_{F}}{\pi^{2} N^{2}\left(\epsilon_{F}\right)} \Sigma_{l} \frac{2(l+1) \sin ^{2}\left(\delta_{l+1}-\delta_{l}\right) N_{l} N_{l+1}}{N_{l}^{(1)} N_{l+1}^{(1)}},
$$

where $\delta_{l}$ are scattering phase shifts, $N_{l}\left(\epsilon_{F}\right)$ is the $l^{\text {th }}$ component of the DOS per spin, and $N_{l}^{(1)}$ is the free-scatter DOS,

$$
N_{l}^{(1)}=\frac{\sqrt{ } \epsilon_{F}}{\pi}(2 l+1) \int_{0}^{R_{s}} u_{l}^{2}\left(r, \epsilon_{F}\right) r^{2} d r .
$$

The scattering phase shifts $\delta_{l}$ were calculated from the radial wave functions $u_{l}$, spherical Bessel functions $j_{l}$ and Neumann functions $n_{l}$ at the muffin-tin radius $R_{s}$ from the formula

$$
\frac{u^{{ }}{ }_{l}\left(r, \epsilon_{F}\right)}{u_{l}\left(r, \epsilon_{F}\right)}=\left.\frac{j^{{ }}{ }_{l}(k r)-n^{{ }}{ }_{l}(k r) \tan \delta_{l}}{j_{l}(k r)-n_{l}(k r) \tan \delta_{l}}\right|_{r=R_{s}},
$$

where the shift energy $\left(E_{f-s h i f t, v o l=i}\right)$ was added to the Fermi energy of the $4^{\text {th }}$ component $1=3$ in both Eqns. 7 and 8. Equation 6 is used with touching MT spheres to minimize errors due to excess interstitial volume as demonstrated in Ref. 26, 27, and 13. The APW total energy results were used to calculate the pressure variation of the bulk moduli B. These values were used to determine the pressure variation of the Debye temperature $\theta_{D}$, which has no experimentally measured bcc equilibrium $\theta_{D}$ below a temperature of $100 \mathrm{~K},{ }^{23}$ using the formula by Moruzzi et al.:24

$$
\theta_{D}=213.4 \sqrt{ } \frac{r_{o} B}{M},
$$

where $B$ is the bulk modulus (in GPa), $r_{o}$ is the Wigner-Seitz radius in Bohr units, and $M$ is the atomic mass. The approximate average phonon frequency $<\omega^{2}>$ is determined as

$$
<\omega^{2}>=\frac{1}{2} \theta_{D}^{2}
$$

and is used both in the denominator of Eq. 4 and in the prefactor of the McMillan equation for the determination of the superconductivity transision temperature $T_{c}, 25$

$$
T_{c}=\frac{<\omega>}{1.2} \exp \left[\frac{-1.04(1+\lambda)}{\lambda-\mu *(1+0.62 \lambda)}\right],
$$

where the Coulomb pseudopotential $\mu *$ was fixed at 0.13 for all three structures.

From our total energy and density of states calculations we determined the input parameters for the $T_{c}$ calculations for the shifted $4 \mathrm{f}$-states for the bcc, hcp, and fcc structures. The bulk modulus for Eu bcc is shown in Figure 9a as a function of pressure. The average phonon frequencies show a similar trend, as displayed in Figure $9 \mathrm{~b}$, since they are derived from the bulk moduli. These trends, where B is directly related to $\langle\omega\rangle$, also agree qualitatively with the proportionality relations used for the lighter elements of fcc yttrium, 26 fcc scandium,, 27 and lanthanum. ${ }^{13}$ 

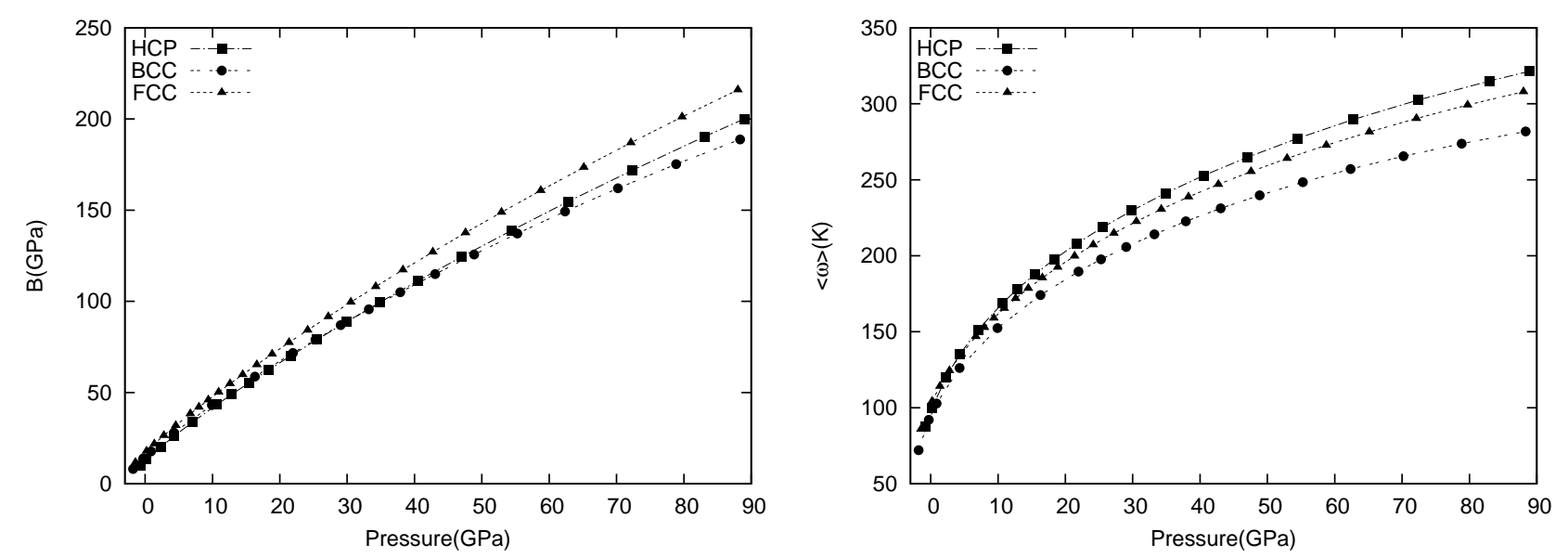

FIG. 9: Bulk modulus B and average phonon frequency $\langle\omega\rangle$ of hcp (solid-line, $\mathbf{\square})$, bcc (dashed-line, $\bullet$ ) and fcc (dash-dot-line, $\mathbf{\Lambda}$ ) as functions of pressure.

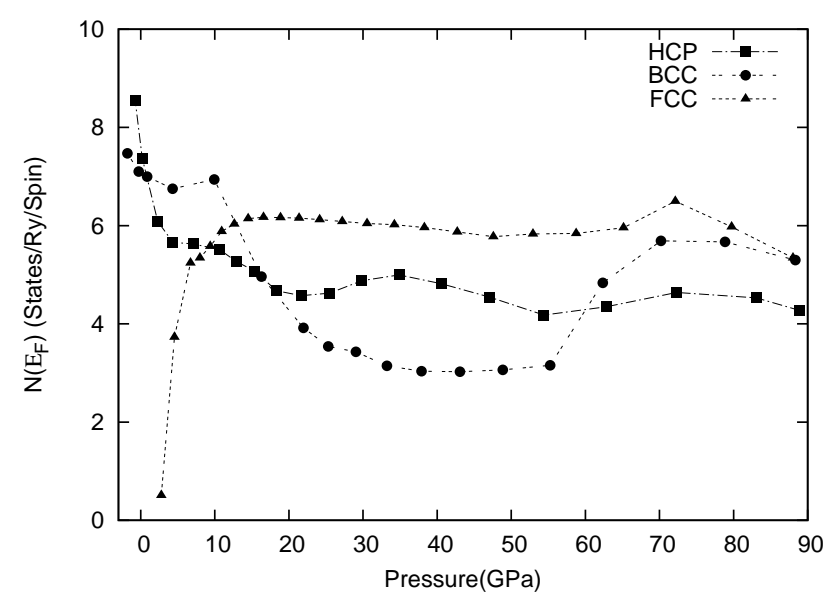

(a) $\mathrm{N}\left(\epsilon_{F}\right)$

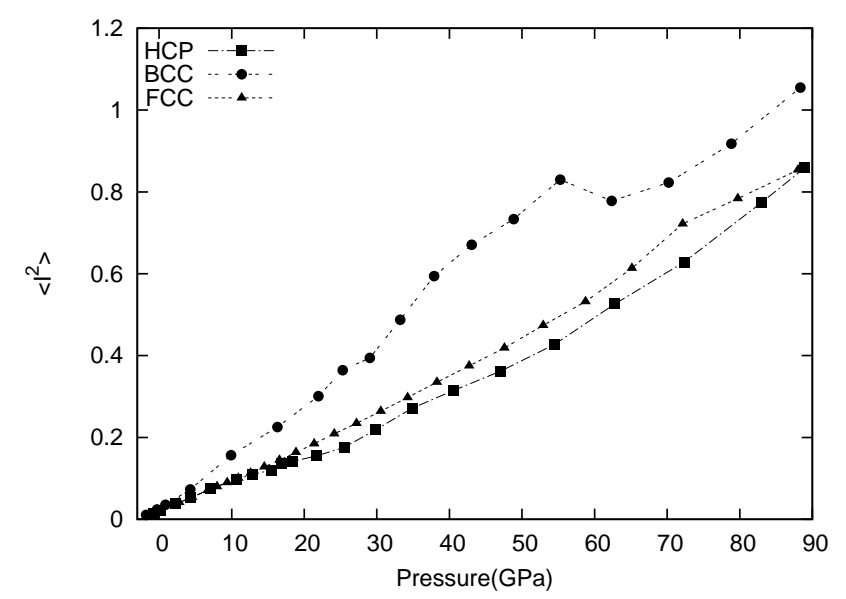

(b) $<I^{2}>$

FIG. 10: Total DOS per spin at $\epsilon_{F}$ and electron-ion matrix element $<I^{2}>$ of hcp (solid-line, $\boldsymbol{\square}$ ), bcc (dashed-line, $\bullet$ ) and fcc (dash-dot-line, $\mathbf{\Lambda})$ as functions of pressure.
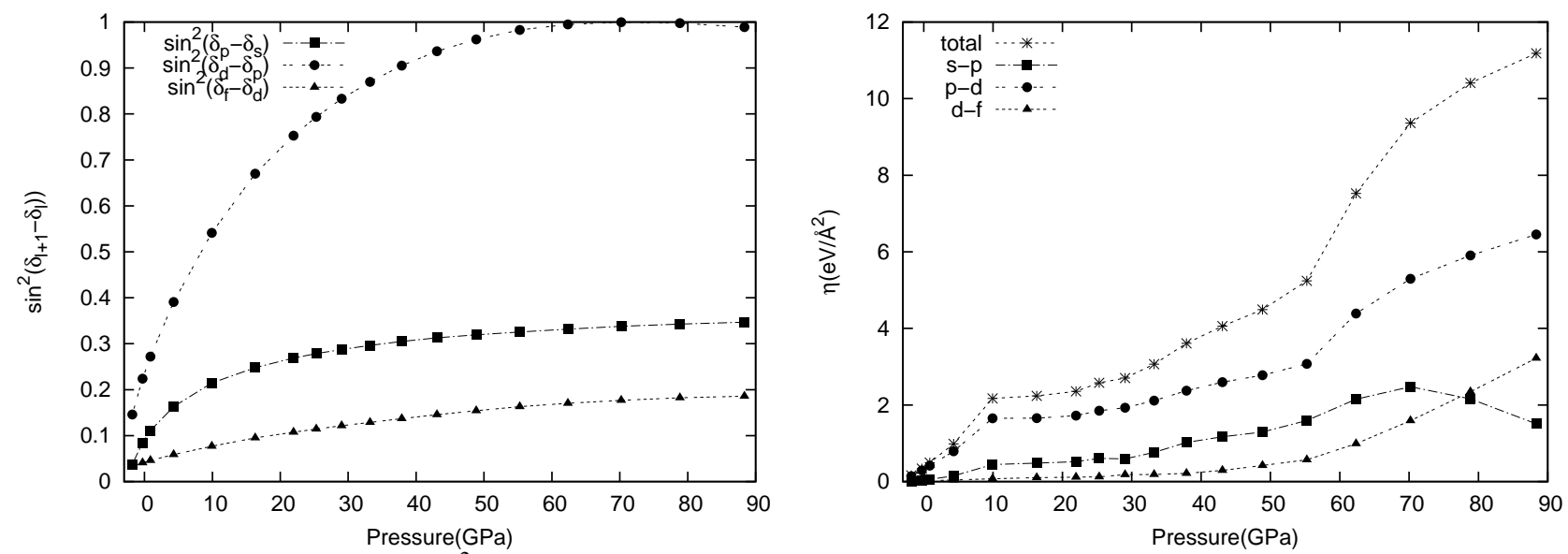

FIG. 11: The phase shift factor $\sin ^{2}\left(\delta_{l+1}-\delta_{1}\right)$ for $l=0$ (squares), $l=1$ (circles), and $l=2$ (triangles) and the total and decomposed Hopfield parameter $\eta$ of bcc europium as functions of pressure.

The density of states at the Fermi level $\mathrm{N}\left(\epsilon_{F}\right)$, electron-ion matrix element $\left\langle I^{2}\right\rangle$, bcc phase shift factor, and bcc total and decomposed Hopfield parameter $\eta$ as functions of pressure are shown in Figs. 10a, 10b, 11a, and 11b, respectively. 


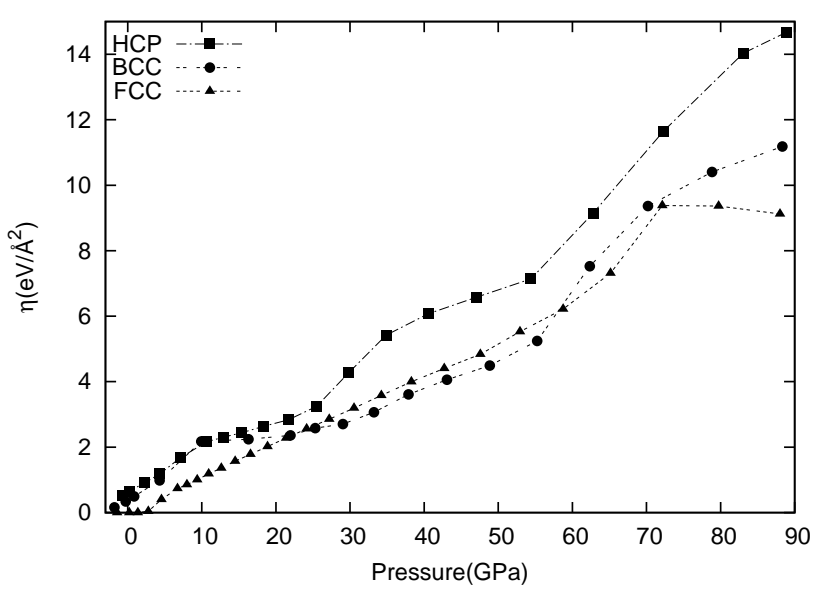

(a) $\eta$

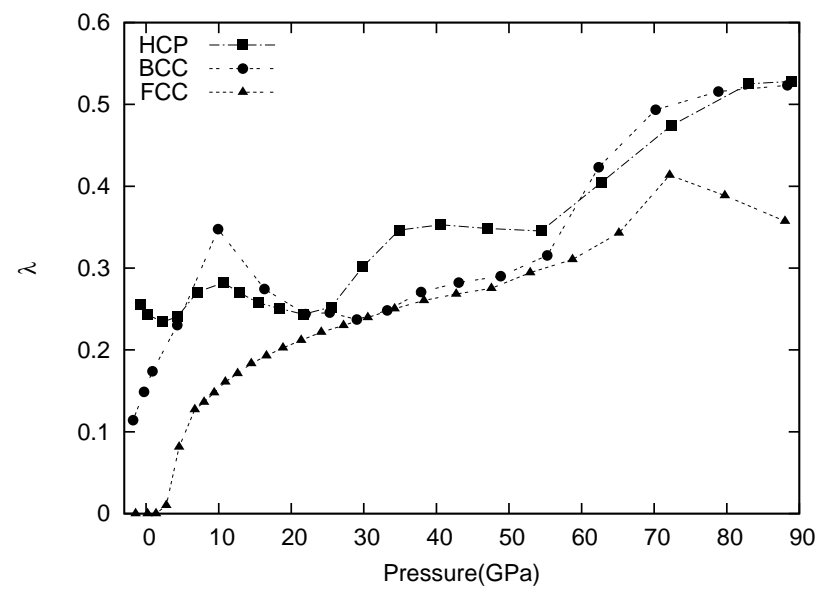

(b) $\lambda$

FIG. 12: Hopfield parameter $\eta$ and electron-phonon coupling constant $\lambda$ of hcp (solid-line, $\mathbf{\square})$, bcc (dashed-line, $\bullet$ ) and fcc (dash-dot-line, $\mathbf{\Lambda}$ ) as functions of pressure.

After about $20 \mathrm{GPa}$ the density of states varies slowly with pressure to $90 \mathrm{GPa}$ for the hcp and fcc. The bcc N $\left(\epsilon_{F}\right)$ increase rapidly after about $55 \mathrm{GPa}$ from near 3.7 states/Ry/spin to almost 5.8 states/Ry/spin near $70 \mathrm{GPa}$. The hcp and fcc $\mathrm{N}\left(\epsilon_{F}\right)$ also decrease after $70 \mathrm{GPa}$.

Although the $\mathrm{N}\left(\epsilon_{F}\right)$ vary slowly with pressure, the electronion matrix element $\left.<I^{2}\right\rangle$ in Eqn. 5 increases rapidly with pressure in all three structures. The increase of the electronion matrix element $<I^{2}>$ comes primarily from the large p-d phase shift factor which is near one at high pressures as shown in Fig. 11a for bcc. Figure 11b shows the bcc total and decomposed Hopfield parameter as a function of pressure. The large increase in total $\eta$ with pressure comes from the large $\mathrm{p}-\mathrm{d} \eta$ contribution. The hcp and fcc also have large $\mathrm{p}-\mathrm{d}$ phase factor and $\eta$ components that increase the total $\eta$.

Figures $12 \mathrm{a}$ and $12 \mathrm{~b}$ respectively show the total $\eta$ and electron-phonon coupling constant $\lambda$ as function of pressure for the hcp, bcc, and fcc. The increasing $\eta$ values in the numerator of Eqn. 4 along with moderate phonon frequencies in the denominator help explain the increase of the electronphonon coupling constant $\lambda$ with pressure. Above $55 \mathrm{GPa}$ the bcc and hcp $\lambda$ goes above 0.4 producing an increase in the superconductity transistion temperature $T_{c}$ shown in Fig. 13. The calculated bcc and hcp $T_{c}$ flattens near $80 \mathrm{GPa}$ because the $\lambda$ levels off at about 0.53 near the same pressure range. The fcc shows a peak in $T_{c}$ near $70 \mathrm{GPa}$ but decreases at higher pressures due to the decreasing fcc $\eta$ that yields smaller $\lambda$ with pressure. 


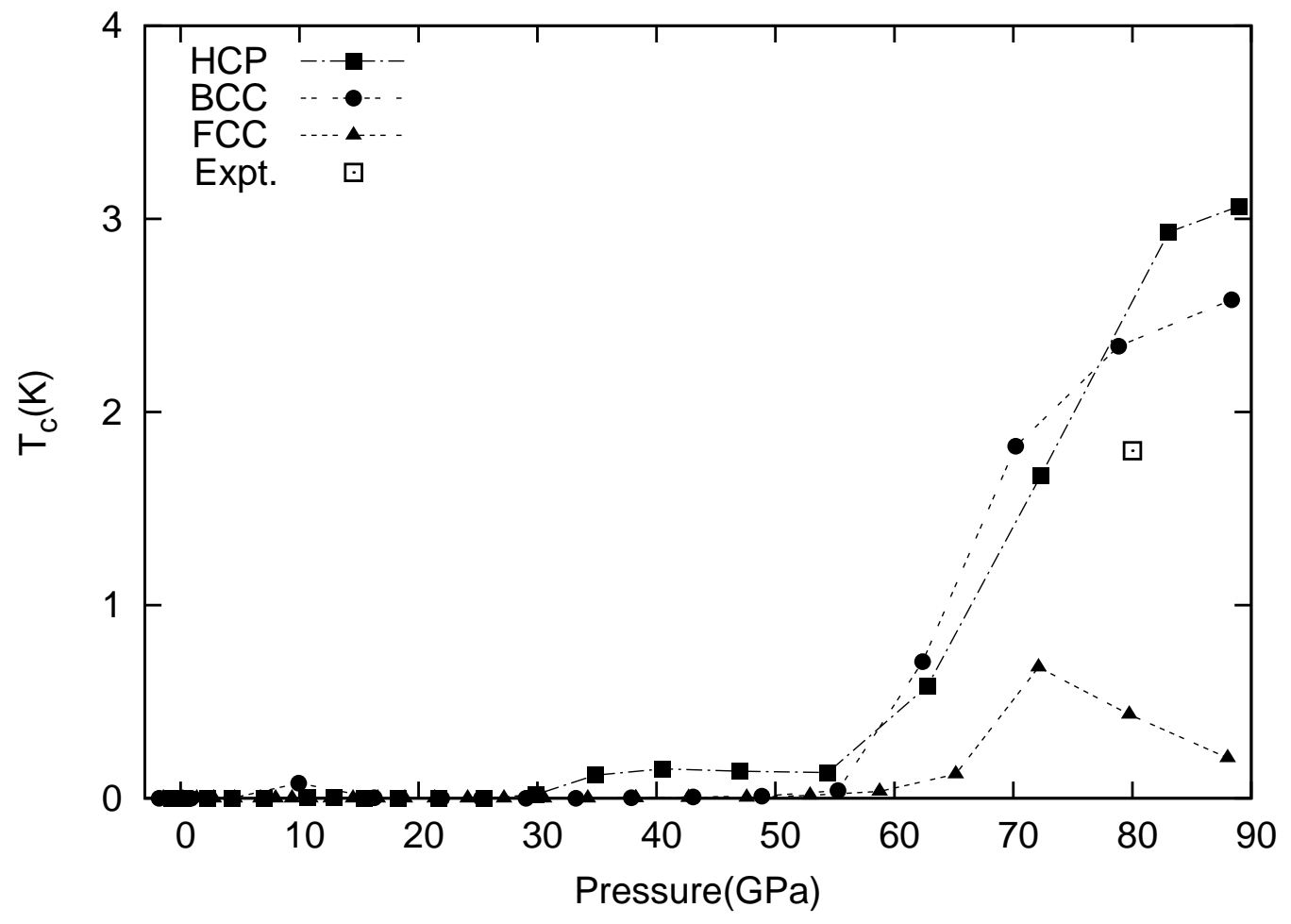

FIG. 13: Transition temperature $T_{c}$ of hcp, bcc, and fcc with $\mu *=0.13$ as functions of pressure. Experimental data from Debessai et al. (Ref.11). 


\section{CONCLUSIONS}

In conclusion, the electronic structure and superconductivity properties of europium have been studied as functions of pressure using the Hedin-Lundqvist LDA form of the exchange-correlation and shifted 4f-band energies within the APW method. In particular, we investigated the total energies for the bcc, fcc, and hcp structures; the density of states, for the bcc and hcp structures and the band structure for the bcc and hcp; and the superconducting properties for the bcc and hcp structures through high pressures, using the RMTA.

On the basis of our total energy calculations, the bcc lattice has been shown to be the equilibrium structure, while the hcp becomes favorable at high pressures. Our density of states calculations agree well with previous results for the bcc structure. Our calculations of the superconducting properties show a gradual increase of the electron-phonon coupling constant to values around 0.5 , reproducing the onset of superconductivity at pressures between 60 and $90 \mathrm{GPa}$.

\section{ACKNOWLEDGEMENTS}

LWN was supported by a National Science Foundation GK12 Fellowship, George Mason University, under grant DGE 0638680. This work was also partially supported by NIST grant 70NANB7H6138.
${ }^{1}$ M. Debessai, T. Matsuoka, J. J. Hamlin, J. S. Schilling, and K. Shimizu, Phys. Rev. Lett. 102, 197002 (2009).

${ }^{2}$ K. Takemura and K. Syassen, J. Phys. F 15, 543 (1985).

3 A. J. Freeman and J. O. Dimmock, Bull. Am. Phys. Soc. 11, 216 (1966).

${ }^{4}$ O. K. Andersen and T. L. Loucks, Phys. Rev. 167, 551 (1968).

5 S-I Kobayasi, M. Fukuchi, and S. Nagai, Solid State Commun. 20, 589 (1976).

${ }^{6}$ M. Matsumoto, I. Shibata, Y. Sakizi, and S-I Kobayasi, J. Phys. Soc. Jpn. 49, 1030 (1980).

${ }^{7}$ M. Matsumoto, M. Fukuchi, Y. Sakizi, and S-I Kobayasi, KEIO Science and Techonology Reports 3497 (1981).

8 M. Matsumoto, I. Shibata, Y. Sakizi, and S-I Kobayasi, J. Phys. F: Met. Phys. 13, 1457 (1983).

9 B. I. Min, H. J. F. Jansen, T. Oguchi, and A. J. Freeman, J. Magn. and Magn. Mater 59, 277 (1986).

10 I. Turek, J. Kudrnovsky, M. Divis, P. Franek, G. Bihlmayer, and S. Blügel, Phys. Rev. B 68, 224431 (2003).

11 J. Kuneš and R. Laskowski, Phys. Rev. B 70, 174415 (2004).

12 L. Hedin and B. L. Lundqvist, J. Phys. C 4, 2064 (1971).

${ }^{13}$ L. W. Nixon, D. A. Papaconstantopoulos, and M. J. Mehl, Phys. Rev. B 78, 214510 (2008).
14 N. G. Nereson, C. E. Olsen, and G. P. Arnold, Phys. Rev. 135, A176 (1964).

15 C. Kittel, Introduction to Solid State Physics, 8 th ed. (John Wiley \& Sons, Inc., New York 2005), pps. 20 and 52.

16 W. A. Grosshans and W. B. Holzapfel, Phys. Rev. B 45, 5171 (1992).

17 G. D. Gaspari and B. L. Gyorffy, Phys. Rev. Lett. 28, 801 (1972).

18 W. H.Butler, J. J.Olson, J. S. Faulkner, and B. L. Gyorffy, Phys. Rev. B 14, 3823 (1976).

19 G. Lehmann and M. Taut, Phys. Status Solidi 54, 469 (1972).

20 M. J. Gillan, J. Phys. Condensed Matter 1, 689 (1989).

${ }^{21}$ M. Richter, J. Phys. D 31, 1017 (1998).

22 J. J. Hopfield, Phys. Rev. 186, 443 (1969).

23 O. V. Lounasmaa, Phys. Rev. 2,143 (1966).

24 V. L. Moruzzi, J. F. Janak, and K. Schwarz, Phys. Rev. B 37, 790 1988.

25 W. L. McMillan, Phys. Rev. 167, 331 (1968).

26 S. Lei, D. A. Papaconstantopoulos, and M. J. Mehl, Phys. Rev. B 75, 024512 (2007).

${ }^{27}$ L. W. Nixon, D. A. Papaconstantopoulos, and M. J. Mehl, Phys. Rev. B 76, 134512 (2007). 